\title{
Influence of Substitution and Milling on Structural and Magnetic Properties of Selected $\mathrm{Sm}\left(\mathrm{Ni}_{1-x} \mathrm{Co}_{x}\right)_{3}$ Compounds
}

\author{
M. Sikora ${ }^{a, b, *}$, D. Blachliński ${ }^{a, b}$, K. OciepkA ${ }^{a, b}$, K. Prusik $^{b, c}$, A. Bajorei $^{a, b}$ \\ AND G. CheŁKOWSKA ${ }^{a, b}$ \\ ${ }^{a}$ A. Chełkowski Institute of Physics, University of Silesia, Uniwersytecka 4, 40-007 Katowice, Poland \\ ${ }^{b}$ Silesian Center for Education and Interdisciplinary Research, 75 Pułku Piechoty 1A, 41-500 Chorzów, Poland \\ ${ }^{c}$ Institute of Materials Science, University of Silesia, 75 Pułku Piechoty 1A, 41-500 Chorzów, Poland
}

The influence of substitution of Co for $\mathrm{Ni}$ in $\mathrm{Sm}\left(\mathrm{Ni}_{1-x} \mathrm{Co}_{x}\right)_{3}$, where $x=0.0,0,1,0.2$, on the structural and magnetic properties has been investigated. Samples with $x=0.0$ and 0.2 were subjected to grinding in a high energy ball mill. The presence of a $\mathrm{PuNi}_{3}$ type of crystal phase was evidenced for bulk crystalline and powders specimens. With an increase in the cobalt content, an increase at $T_{c}$ was observed. From the magnetic isotherms, the magnetic entropy changes were determined both for solid and powdered samples. It has been shown that the value of the magnetic entropy change at $T_{c}$ is doubled for the bulk sample with $x=0.2$ but it has decreased significantly for all powdered samples.

DOI: 10.12693/APhysPolA.133.486

PACS/topics: 75.30.Cr, 75.30.Sg, 75.50.Gg, 75.50.Vv

\section{Introduction}

Rare earth and transition metals intermetallic compounds such as alloys were widely studied in previous years because of interesting $3 \mathrm{~d}-4 \mathrm{f}$ effects and a possibility of application in different branches of industry and technique $[1,2]$. Generally, the magnetic properties of rare earth and transition metals depend on crystal structure because the atoms of transition elements have an exposed 3d shell and are susceptible on their nearest surrounding. In recent years, nanocrystalline $\mathrm{RE} / 3 \mathrm{~d}$ alloys have attracted an increasing interest because of their enhanced magnetic parameters while compared to their bulk counterparts $[3,4,5]$. As an example, one can mention a high coercivity of $33 \mathrm{kOe}$ for the nanocrystalline $\mathrm{SmCo}_{3}$ compound. In this paper, we focus on structural and magnetic changes in a $\mathrm{SmNi}_{3}$ compound upon the $\mathrm{Ni} / \mathrm{Co}$ substitution and pulverization to a size of approximately $100 \mathrm{~nm}$.

\section{Experimental}

$\mathrm{Sm}\left(\mathrm{Ni}_{1-x} \mathrm{Co}_{x}\right)_{3}$ samples where $x=0.0,0.1$ and 0.2 were prepared by arc melting of high purity elements (99,99\% purity) under argon atmosphere. An excess 5\% wt. of Sm element was added to overcome the weight loss during the melting. To obtain a high homogeneity of the prepared compounds, all samples were re-melted several times. Afterwards, the as-cast samples were wrapped in tantalum foil, placed in quartz tubes and annealed at $90{ }^{\circ} \mathrm{C}$ for $196 \mathrm{~h}$. Samples of $\mathrm{Sm}\left(\mathrm{Ni}_{1-x} \mathrm{Co}_{x}\right)_{3}$ with the highest purity were powdered in a high energy ball mill Mixer Mill 400 (Retsch) with a frequency of $30 \mathrm{~Hz}$ for $12 \mathrm{~h}$

*corresponding author; e-mail: marcin.sikora.us@gmail.com and $24 \mathrm{~h}$. The grinding procedure, described earlier in [1], was carried out in dimethylformamide (DMF) using Eppendorf vials and $2 \mathrm{~mm}$ balls made of $\mathrm{ZrO}_{2}$; the balls to powder ratio was close to 5:1 by weight. The crystal structure was measured by X-Ray Diffraction method on an Empyrean XRD diffractometer (PANalytlical). All measurements were carried out at room temperature using a Cu X-ray source. Electron Microscopy (SEM) studies were carried out using Jeol JSM 6480 operated at $20 \mathrm{kV}$ after $24 \mathrm{~h}$ of grinding. All examined powders were fixed to the sample holder by the carbon double coated conductive tape. The SEM images were taken in the SE mode in the magnification range of 2000 to 40000 times. All magnetic measurements were performed on a SQUID (superconducting quantum interference device) magnetometer MPMS XL7 (Quantum Design). Temperature measurements were conducted in the range of $2 \mathrm{~K}$ to $400 \mathrm{~K}$. The magnetic field was being changed up to $7 \mathrm{~T}$.

\section{Results and discussion}

\subsection{Crystal structure}

Crystal structures of all measured samples were refined using the Rietveld method. The match function used to analysis and approximation was a Pseudo-Voigt function with UWV parameters (using the FullProf program). The analysis showed that all the samples crystallized in a $\mathrm{PuNi}_{3}$-type of structure ( $R$ - $3 m$ space group). For the sample with $x=0.1$, the diffraction patterns showed a presence of an additional phase $\mathrm{SmNi}_{2}$, around $3 \%$ by weight of the whole sample. The example XRD pattern of $\mathrm{Sm}\left(\mathrm{Ni}_{0.8} \mathrm{Co}_{0.2}\right)_{3}$ is showed in Fig.1. The cell parameters change from $a=5.0040 \pm 0.0009 \AA$ and $c=24.6223 \pm 0.0026 \AA$ for $\mathrm{SmNi}_{3}$ to $a=5.0130 \pm 0.0123 \AA$ and $c=24.6648 \pm 0.0151 \AA$ for $\mathrm{Sm}\left(\mathrm{Ni}_{0.8} \mathrm{Co}_{0.2}\right)_{3}$. The character of these changes is linear. This increase in the cell parameters is due to the difference in atomic radii of 
nickel and cobalt. Cobalt has a greater atom radius than nickel, causing an extension of the primitive cell. With the increase of the milling time, a gradual amorphization was observed (Fig. 1).

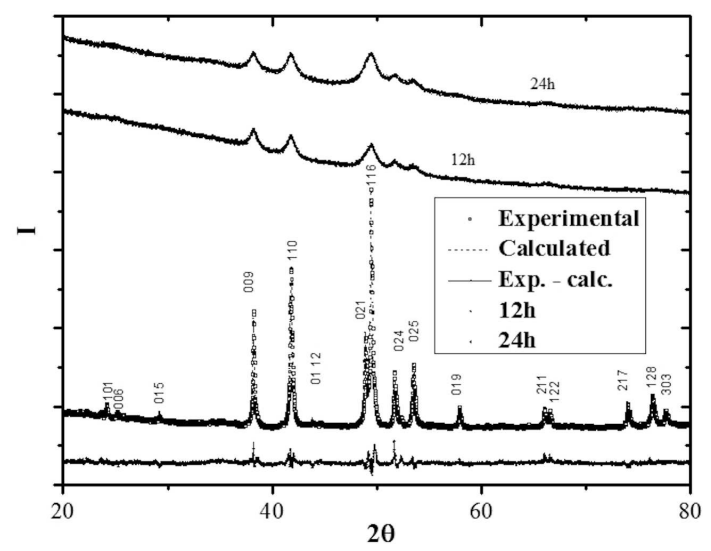

Fig. 1. XRD diffraction patterns for bulk $\mathrm{Sm}\left(\mathrm{Ni}_{0.8} \mathrm{Co}_{0.2}\right)_{3}$ and vanishing of the crystal structure of ground $\mathrm{Sm}\left(\mathrm{Ni}_{0.8} \mathrm{Co}_{0.2}\right)_{3}$.

The values of the crystallites size determined by the use of the Scherrer's equation [6] are reduced by milling for the two synthesized samples. However, for pure $\mathrm{SmNi}_{3}$, this parameter varies from $68.87 \pm 3.50 \mathrm{~nm}$ (bulk sample) to $20.29 \pm 1.40 \mathrm{~nm}(24 \mathrm{~h})$, whereas for the $x=0.2$, it is reduced from $45.02 \pm 2.30 \mathrm{~nm}$ (bulk sample) to $15.80 \pm$ $0.80 \mathrm{~nm}(24 \mathrm{~h})$, respectively. It is worth mentioning that the value of the obtained crystallites is smaller than that obtained from SEM images, because the crystallites size is not synonymous with the particles size, which is visible on SEM picture (see Fig. 2).

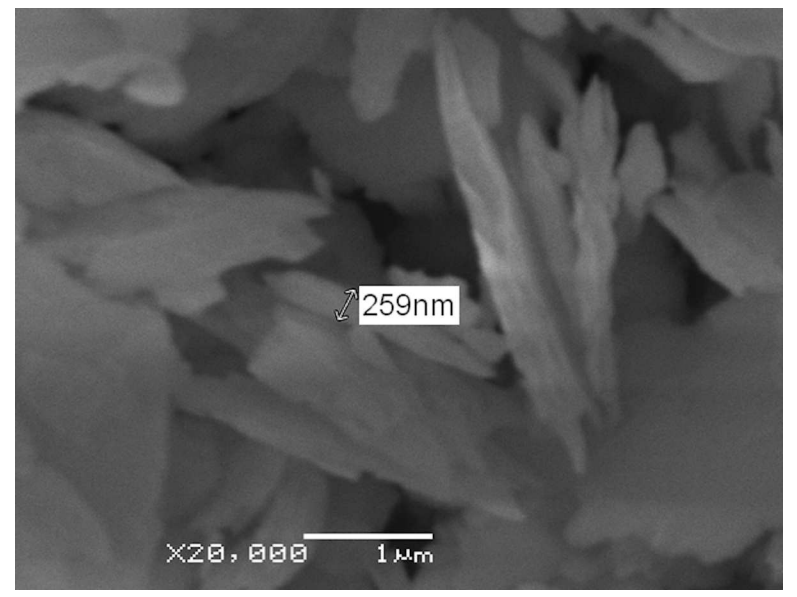

Fig. 2. SEM image obtained for the $\mathrm{SmNi}_{3}$ compound.

\subsection{Magnetic properties}

The temperature dependence of magnetization $M(T)$ has been measured at the applied field of $0.1 \mathrm{~T}$. The temperature of phase transition $T_{c}$ for each sample has been estimated from $\mathrm{d} M_{F C} / \mathrm{d} T$ as the minimal value. The values of $T_{c}$ are shown in Fig. 3. It was observed that an increase in the Co content is accompanied by a rapid increase in the values of $T_{c}$ and magnetization. The growth of $T_{c}$ may be caused by changes in magnetic interactions between atoms as a result of doping. Interactions in these compounds are mainly derived from those within the $3 d-3 d$ band of nickel/cobalt and $3 d-4 f$ band of nickel/cobalt and samarium. The increase in the magnetization with the $\mathrm{Ni} / \mathrm{Co}$ substitution may be related to the fact that Co has a higher magnetic moment than the Ni atom. In the magnetically ordered state, all compounds exhibit remarkable thermomagnetic irreversibility (Fig. 3). In the field cooled (FC) mode, the $M(T)$ dependence shows an increase at low temperatures until saturation. However, in the zero field cooled (ZFC) mode, the $M(T)$ curves are quite different. In all $M_{Z F C}(T)$ curves, the maxima are visible, after which the values of $M_{Z F C}(T)$ fall to zero at low temperatures. The unusual shape of the ZFC curves as well as the large FC-ZFC difference may be related to a high magnetic anisotropy of these compounds. For $\mathrm{SmNi}_{3}$ and $\mathrm{Sm}\left(\mathrm{Ni}_{0.9} \mathrm{Co}_{0.1}\right)_{3}$, a small negative magnetization $M_{Z F C}(T)$ was observed at low temperatures. It may be caused by the trapped field in a superconducting magnet. This phenomenon and its mechanism are briefly described in Kumar's article [7]. The magnetocaloric effect (MCE) was evaluated using the formula (1), based on a family of magnetic isotherms $M(H)$ measured at different temperatures:

$$
\Delta S_{M}\left[H_{m}, T\right]=\int_{0}^{H_{m}}\left(\frac{\partial M}{\partial T}\right)_{H} \mathrm{~d} H,
$$

where $\Delta S_{M}[H, T]$ is the magnetic entropy change at a temperature $T$ due to a magnetic field change from 0 to $H_{m}$.

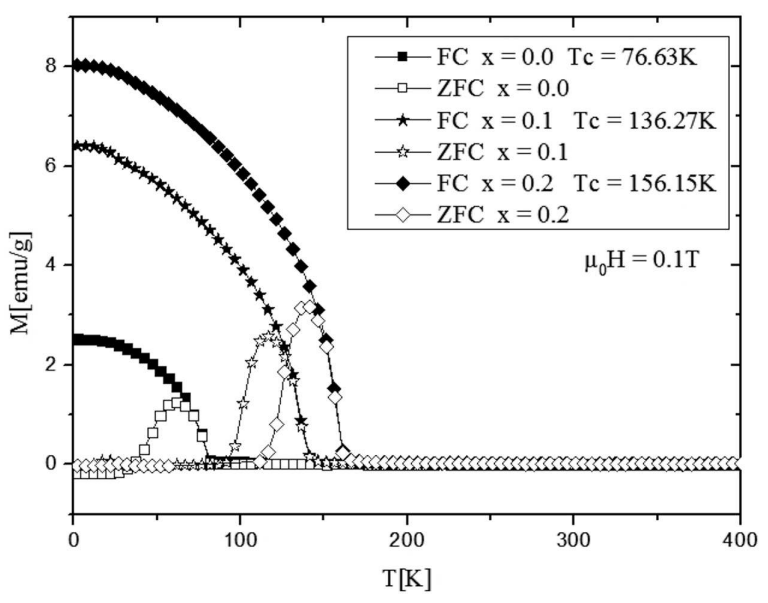

Fig. 3. FC/ZFC magnetization curves of $\mathrm{Sm}\left(\mathrm{Ni}_{1-x} \mathrm{Co}_{x}\right)_{3}$.

In Fig. 4, the magnetocaloric effect of $\mathrm{SmNi}_{3}$ and $\mathrm{Sm}\left(\mathrm{Ni}_{0.8} \mathrm{Co}_{0.2}\right)_{3}$ is presented for the bulk form as well as after $24 \mathrm{~h}$ of grinding. It is observed that the replacement of nickel by cobalt increases the value of the magnetocaloric effect. In the field of $2 \mathrm{~T}$, the value of 
magnetic entropy changes from $0.188 \mathrm{~J} / \mathrm{kg} \mathrm{K}$ for $\mathrm{SmNi}_{3}$ to $0.367 \mathrm{~J} / \mathrm{kg} \mathrm{K}$ for $\mathrm{Sm}\left(\mathrm{Ni}_{0.8} \mathrm{Co}_{0.2}\right)_{3}$. Furthermore with the increase of the magnetic field, the values of entropy changes grow linearly. As expected, the maximum value of entropy decreases with progressive time of milling, which is observed in other papers $[1,2,8]$. In $\mathrm{SmNi}_{3}$, the position of the MCE maximum changes from $76 \mathrm{~K}$ for bulk to $60 \mathrm{~K}$ for the milled sample. This effect may be related to the weakening of the interactions between the magnetic particles with grinding. No similar shift for $\mathrm{Sm}\left(\mathrm{Ni}_{0.8} \mathrm{Co}_{0.2}\right)_{3}$ was observed. From $M(H)$ curves, the saturation magnetization $M_{s}$ and the coercivity $H_{c}$ were obtained. Chemical substitution and milling have a big impact on the values of $M(H)$ and $H_{c}$. The coercivity dropped from $5.53 \mathrm{~T}$ for $\mathrm{SmNi}_{3}$ to $0.27 \mathrm{~T}$ for $\mathrm{Sm}\left(\mathrm{Ni}_{0.8} \mathrm{Co}_{0.2}\right)_{3}$. The saturation $M_{s}(x)$ also has changed with the replacement of $\mathrm{Ni}$ by Co having a greater magnetic moment and it increased from $0.06 \mu_{\mathrm{B}} / \mathrm{fu}$ for $x=0.0$ to $0.106 \mu_{\mathrm{B}} / \mathrm{fu}$ for $x=0.2$. The pulverization of the samples caused an extreme decrease in the $H_{c}$ and an increase in the $M_{s}$ value.

TABLE I

Values of magnetic parameters of the examined materials.

\begin{tabular}{c|c|c|c|c|c}
\hline \hline$x$ & $\begin{array}{c}T_{c} \\
{[\mathrm{~K}]}\end{array}$ & $\begin{array}{c}-\Delta S_{M}(5 \mathrm{~T}) \\
{[\mathrm{J} / \mathrm{kg} \mathrm{K}]}\end{array}$ & $\begin{array}{c}\mu_{\text {eff }} \\
{\left[\mu_{\mathrm{B}} / \mathrm{fu}\right]}\end{array}$ & $\begin{array}{c}H_{c}(2 \mathrm{~K}) \\
{[\mathrm{T}]}\end{array}$ & $\begin{array}{c}M_{s}(2 \mathrm{~K}) \\
{\left[\mu_{\mathrm{B}} / \mathrm{fu}\right]}\end{array}$ \\
\hline 0.0 & 76 & 0.188 & 0.041 & 5.53 & 0.060 \\
0.1 & 136 & - & 0.061 & 1.03 & 0.069 \\
0.2 & 156 & 0.367 & 0.091 & 0.270 & 0.106 \\
$0.0(24 \mathrm{~h})$ & 60 & 0.069 & - & 0.135 & 0.216 \\
$0.2(24 \mathrm{~h})$ & 156 & 0.102 & - & 0.130 & 0.352
\end{tabular}

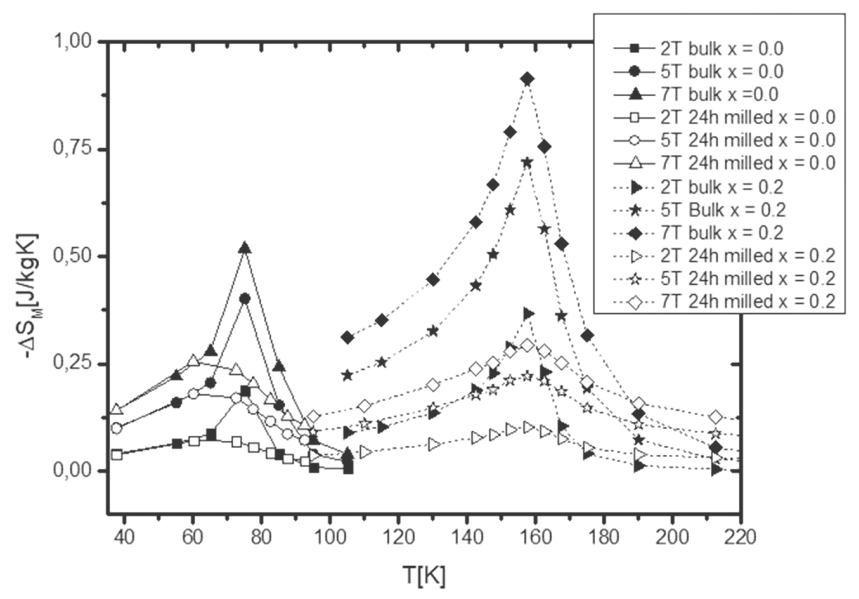

Fig. 4. Magnetic entropy changes of bulk and powder $\mathrm{Sm}\left(\mathrm{Ni}_{1-x} \mathrm{Co}_{x}\right)_{3}$.

Applying the modified Curie-Weiss law to bulk samples in paramagnetic state, the effective magnetic moments $\mu_{e f f}$ were obtained. An increase in the value of the effective moment $\mu_{e f f}$ with the increase in the cobalt concentration has been observed. The values were $0.041 \mu_{\mathrm{B}} / \mathrm{fu}$, $0.061 \mu_{\mathrm{B}} / \mathrm{fu}$ and $0.091 \mu_{\mathrm{B}} /$ fu for $x=0.0,0.1$ and 0.2 , respectively, but they are much smaller than the theoretical value of a free $\mathrm{Sm}^{3+}$ ion. It may suggest that nickel has a magnetic moment equal to $0.27 \mu_{\mathrm{B}}$ and this moment is oriented antiparallel to the samarium ion moment. Comparing our results with similar systems such as $\mathrm{RT}_{2}$ and $\mathrm{RT}_{3}$ where $\mathrm{R}=\mathrm{Gd}, \mathrm{Sm}$, we can see that the $\mathrm{Ni}$ atoms have a nonzero value of the magnetic moment between $0.15 \mu_{\mathrm{B}}$ to $0.3 \mu_{\mathrm{B}}$ oriented antiparallel to the rare earth moment [9-11]. The obtained parameters are listed in Table I.

\section{Conclusions}

We have examined a series of $\mathrm{Sm}\left(\mathrm{Ni}_{1-x} \mathrm{Co}_{x}\right)_{3}$ compounds with $x=0.0,0.1,0.2$ for their general structural and magnetic properties with an emphasis on the magnetocaloric effect. The chemical substitution and pulverization have a significant impact on the basic physical properties. The substitution of Ni by Co causes an increase in the lattice parameters $a$ and $c$, the effective moment $\mu_{e f f}$, the saturation magnetization $M_{s}$, and the entropy change $-\Delta S_{M}$. Simultaneously, an increase in the Co concentration causes a rapid decrease of the coercivity $H_{c}$. The low values of $\mu_{e f f}$ and $M_{s}$ suggest the $\mathrm{Ni}$ has a magnetic moment $\mu_{\mathrm{Ni}}$ equal to $0.27 \mu_{\mathrm{B}}$ oriented antiparallel to the Sm moment. A chemical modification consisting of substitution of $\mathrm{Ni}$ by Co has enhanced the magnetocaloric properties of the examined materials. The value of the magnetic entropy changes increased approximately two-fold for the doped bulk sample while compared with the undoped one. Mechanical grinding leads to an increase in the saturation and a decrease in the coercivity in all examined samples, together with a great drop of the magnetic entropy changes at the phase transition temperature $T_{c}$.

\section{References}

[1] A. Bajorek, P. Skornia, K. Prusik, M. Wojtyniak, G. Chełkowska, Mater. Charact. 101, 58e70 (2015).

[2] A. Chrobak, A. Bajorek, G. Chełkowska, G. Haneczok, M. Kwiecień, Phys. Status Solidi A 206, 731 (2009).

[3] O. Gutfleisch, A. Bollero, A. Handstein, D. Hinz, A. Kirchner, A. Yan, K. H. Müller, L. Schultz, J. Magn. Magn. Mater. 242, 1277 (2002).

[4] C. D. Milham, J. App. Phys. 75, 5659 (1994).

[5] X. Song, N. Lu, M. Seyring, M. Rettenmayr, W. Xu, Z. Zhang, J. Zhang, Appl. Phys. Lett. 94, 023102 (2009).

[6] A. Monshi, M. R. Foroughi, M. R. Monshi, World J. Nanosc. Eng. , 154 (2012.

[7] N. Kumar, A. Sundaresan, Solid State Comm. 150, 1162 (2010).

[8] M.H. Phan, M.B. Morales, C.N. Chinnasamy, B. Latha, V.G. Harris, H. Srikanth, J. Phys. D: Appl. Phys. 4, 115007 (2009).

[9] K. Yano, I. Umehara, T, Miyazawa, Y. Adachi, K. Sato, Physica B 367, 81 (2005).

[10] M. Kwiecień, G. Chełkowska, Molecular Physics Reports 40, 108 (2004).

[11] J. Farrell, W.E. Wallace, Inorg. Chem 5, 105 (1966). 